\title{
The majority of conversion total hip arthroplasties can be considered primary replacements - a matched cohort study
}

\author{
Georges Vles, Luke Simmonds, Mark Roussot, Andrea Volpin, Fares Haddad, Sujith Konan \\ Department of Trauma and Orthopaedics, University College London Hospitals, London, United Kingdom
}

The success of conversion Total Hip Arthroplasty (THA) among primary THA and revision THA remains unclear. We hypothesized that most conversion THAss can be performed using primary implants and will have an uncomplicated post-operative course. Thirty-six patients ( 23 females, mean age $68,0 y$ ) who underwent conversion THA for failed interventions for proximal femur fractures in the period 2006-2018 were matched sequentially against patients of the same sex and age who underwent primary THA or revision THA. Data was collected on implants used, major complications, and mortality. PROMs used included the Western Ontario and McMaster Osteoarthritis Index, Harris Hip Score, Visual Analogue Scale and the EQ-5D Health Questionnaire. Seventytwo percent of patients who underwent conversion THA were treated with primary implants and never suffered from a major complication. PROMs were excellent for this group of patients. The distinction primary / conversion / revision THA could not explain differences in outcomes, however the necessity of using revision implants and the development of major complications could. The majority of conversion total hip arthroplasties can be considered a primary replacement. Predicting outcomes for THA should focus on patient frailty and technical difficulties dealing with infection, stability and loss of bone stock

Declaration of conflicting interests : GV, LS, MR, AV declare to have no conflict of interest. FSH : Editor in chief : Bone and Joint Journal ; receives royalties from : Smith \& Nephew, Stryker, Corin, MatOrtho; receives institutional and research support from: Smith \& Nephew, Stryker, Corin, MatOrtho, Zimmer Biomet, NIHR, IOC. Sujith Konan's Col, SK - Smith \& Nephew Education - Faculty.

Funding : This research received no specific grant from any funding agency in the public, commercial, or not-for-profit sectors. and should discard the conversion versus revision terminology.

Keywords : Proximal femur fracture; Total Hip Arthroplasty ; Conversion ; Primary ; Revision ; Matched cohort study.

\section{INTRODUCTION}

A primary Total Hip Arthroplasty (pTHA) is most commonly performed for osteoarthritis (OA), usually has an uneventful post-operative course and is known for its excellent long-term results (1).

Georges Vles ${ }^{1,2}$

Luke Simmonds ${ }^{1}$,

Mark Roussot ${ }^{1}$,

Andrea Volpin ${ }^{1}$,

Fares Haddad ${ }^{1}$,

- Sujith Konan ${ }^{1}$

${ }^{1}$ Department of Trauma and Orthopaedics, University

College London Hospitals, London, 235 Euston Rd,

Fitzrovia, London NW1 2BU, United Kingdom.

${ }^{2}$ Division of Orthopaedic Surgery, University Hospitals

Leuven, Gasthuisberg, Herestraat 49, 3000 Leuven, Belgium.

Correspondence : G.F. Vles, Division of Orthopaedic Surgery, University Hospitals Leuven, Herestraat 49, 3000 Leuven, Belgium. Phone : +32 163388 27, Fax : +32 16338824 .

Email : Georges.Vles@uzleuven.be

- 2021, Acta Orthopædica Belgica.

Acta Orthopædica Belgica, Vol. 87 - 1 - 2021 
Figure 1. - Spectrum of failed hardware initially used to treat proximal femur fractures.

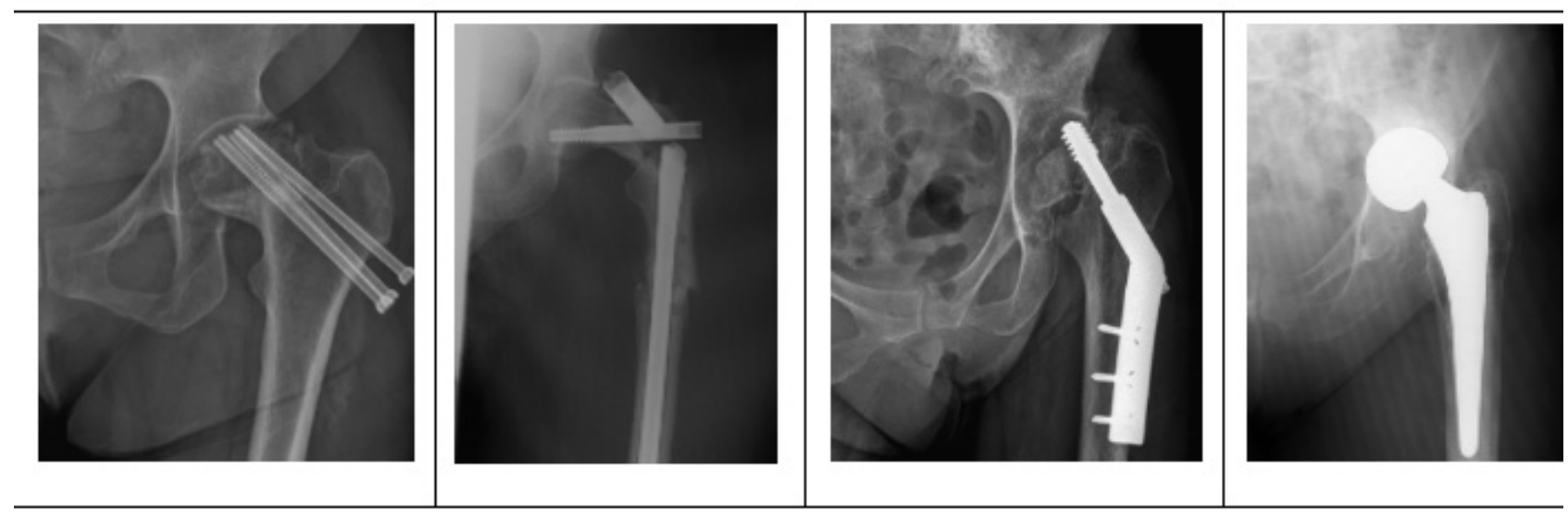

From left to right : avascular necrosis $(\mathrm{AVN})$ of the femoral head after cannulated screw fixation ; varus collaps after fatigue failure of a long cephomedullary nail ; AVN and cut out of a DHS ; protrusion of a HA.

Nevertheless, failures do occur, for a variety of reasons, and are most often followed by a revision (r) THA. This is a technically more demanding procedure, revision implants are often necessary, and can range from changing a wornout polyethylene liner in a not yet unstable hip to a 2-stage revision for a difficult-to-treat infection with substantial bone loss. Results of rTHAs are less favourable than those seen in pTHAs due to the fact that complications are more common, survival of implants is shorter, and patients report lower on outcome measures (PROMs) (2-4).

A third group of hip replacements is made up by the conversion (c) THAs. These are usually performed when an intervention for a proximal femur fracture has failed (figure 1) and is salvaged by THA (5-9). Again, this a very diverse group as it can include patients undergoing placement of an additional cup in a hemi-arthroplasty (HA) that is causing painful erosion of the native acetabulum or patients with infected cephomedullary nails with non-union, significant bone loss and an escaped abductor apparatus. Besides these technical difficulties, there frequently are concomitant medial issues as the typical patient requiring cTHA is of old age, has multiple health issues, and usually has been barely mobile in the period awaiting salvage surgery.

Attempts have been made to determine the success of cTHA among pTHA and rTHA, as this has implications for patient consenting and institutional reimbursement (10-17). It has been suggested that a cTHA should be considered an rTHA, but also that it is a distinct entity with outcomes in between pTHA and rTHA $(13,14,16)$. Interpreting these studies is difficult, as matched cohort analyses are rare, follow-up differs between groups, but most importantly because of the fact that very diverse groups of cTHAs are compared to very diverse groups of rTHAs (10-17). This raises the question whether it is useful to predict outcomes based on this distinction in the first place $(15,16)$. There is a subgroup of patients that undergoes cTHA using primary implants, who will never develop any complications and whose satisfaction probably resemblances that of patients with pTHAs (17).

We therefore performed a matched cohort study and formulated the following three hypotheses :

1. The distinction pTHA / cTHA / rTHA will not be able to explain differences in outcomes.

2. Necessity of revision implants and development of major complications will be able to explain differences in outcomes.

3. Most cTHAs can be performed using primary implants and will have an uncomplicated postoperative course.

\section{MATERIALS AND METHODS}

The theatre diaries of our dedicated hip unit were meticulously searched for patients who underwent cTHA for failed interventions for proximal femur 
Table I. - Demographic data, mortality and PROMs of the three cohorts of hip replacements

\begin{tabular}{|c|c|c|c|c|c|}
\hline & pTHA & cTHA & rTHA & $\mathbf{F}$ & $\mathbf{p}$ \\
\hline $\mathrm{N}$ & 36 & 36 & 36 & & \\
\hline $\operatorname{Sex}(F, \%)$ & $23(63,9 \%)$ & $23(63,9 \%)$ & $24(66,7 \%)$ & & \\
\hline Age fracture (mean) & NA & 63,6 y (SD 14,8; 27-85) & NA & & \\
\hline Age p/cTHA (mean) & 69,1 y (SD 12,4; 39-86) & 68,0 y (SD 14, 0; 34-86) & 59,7 y (SD 14,6; 29-82) & 4,177 & 0,18 \\
\hline Age rTHA (mean) & NA & NA & 69,0 y (SD 12,8; 39-85) & & \\
\hline Side (L, \%) & $14(38,9 \%)$ & $24(66,7 \%)$ & $12(33,3 \%)$ & & \\
\hline ASA (median) & $2(1-3)$ & $2(1-3)$ & $2(1-3)$ & & \\
\hline $\mathrm{F} / \mathrm{U}$ (mean) & 6,4 y (SD 4,3; 1,2-13,2) & 6,4 y (SD 4,3; 1,2-13,2) & 6,4 y (SD 4,3; 1,2-13,2) & 0,000 & 1,00 \\
\hline VAS overall health (mean) & 67,8 (SD 24,7; 20-100) & $61,0($ SD 25,$5 ; 20-100)$ & 65,1 (SD 18,7; 30-99) & 0,428 & 0,65 \\
\hline Major complications & 4 & 4 & 6 & 0,321 & 0,73 \\
\hline 1 year mortality $(\%)$ & $3(8,3 \%)$ & $1(2,8 \%)$ & $0(0 \%)$ & 1,828 & 0,17 \\
\hline Mortality at final F/U (\%) & $9(25,0 \%)$ & $7(19,4 \%)$ & $6(16,7 \%)$ & 0,391 & 0,68 \\
\hline \multicolumn{6}{|l|}{ WOMAC } \\
\hline Pain (mean) & 79,7 (SD 28,9; 20,0-100) & 66,1 (SD 29,8; 0-100) & 72,2 (SD 33,2; 0-100) & 1,036 & 0,36 \\
\hline Stiffness (mean) & $82,2($ SD 28,$4 ; 12,5-100)$ & 64,7 (SD 34,9; 0-100) & $63,2(\mathrm{SD} 33,9 ; 0-100)$ & 2,030 & 0,14 \\
\hline Difficulties (mean) & 69,7 (SD 30,8; 7,4-100) & 59,4 (SD 35,5; 0-100) & $58,1($ SD 28,7; 0-97,1) & 0,746 & 0,48 \\
\hline Total (mean) & $72,7($ SD 29,0; 12,5-100) & $61,8(\mathrm{SD} 32,5 ; 6,3-100)$ & 61,6 (SD 29,1; 0-97,9) & 0,850 & 0,43 \\
\hline \multicolumn{6}{|l|}{ EQ-5D } \\
\hline Mobility (mean) & $1,4(\mathrm{SD} 0,5 ; 1-2)$ & $1,7(\mathrm{SD} 0,7 ; 1-3)$ & $1,8(\mathrm{SD} 0,6 ; 1-3)$ & 2,335 & 0,11 \\
\hline Self-care (mean) & $1,5(\mathrm{SD} 0,6 ; 1-3)$ & $1,5(\mathrm{SD} 0,7 ; 1-3)$ & $1,8(\mathrm{SD} 0,5 ; 1-3)$ & 1,513 & 0,23 \\
\hline Usual activities (mean) & $1,7(\mathrm{SD} 0,7 ; 1-3)$ & $1,7(\mathrm{SD} 0,8 ; 1-3)$ & 1,9 (SD 0,5;s 1-3) & 0,991 & 0,34 \\
\hline Pain (mean) & $1,5(\mathrm{SD} 0,7 ; 1-3)$ & $1,4(\mathrm{SD} 0,6 ; 1-3)$ & $1,8(\mathrm{SD} 0,6 ; 1-3)$ & 1,496 & 0,23 \\
\hline Anxiety (mean) & $1,3(\mathrm{SD} 0,6 ; 1-3)$ & $1,5(\mathrm{SD} 0,7 ; 1-3)$ & $1,4(\mathrm{SD} 0,6 ; 1-3)$ & 0,694 & 0,50 \\
\hline \multicolumn{6}{|l|}{ HHS } \\
\hline Pain & $75,8(\mathrm{SD} 32,3 ; 0-100)$ & 67,6 (SD 31,7; 0-100) & $68,2(\mathrm{SD} 33,4 ; 0-100)$ & 0,396 & 0,68 \\
\hline Limp & 75,1 (SD 30,9; 0-100) & 56,5 (SD 41,4; 0-100) & $52,5(\mathrm{SD} 38,4 ; 0-100)$ & 1,976 & 0,15 \\
\hline Support & $61,2(\mathrm{SD} 37,7 ; 0-100)$ & 41,9 (SD 38,2; 0-100) & 43,4 (SD 37,8; 0-100) & 1,705 & 0,19 \\
\hline Walking distance & $56,0(\mathrm{SD} 32,1 ; 0-100)$ & 45,5 (SD 34,6; 0-100) & $39,9(\mathrm{SD} 29,6 ; 0-100)$ & 1,190 & 0,31 \\
\hline Stairs & $55,3(\mathrm{SD} 34,9 ; 0-100)$ & 45,7 (SD 32,6; 0-100) & 37,5 (SD 30,0;0-100) & 1,378 & 0,26 \\
\hline Socks and shoes & $63,2(\mathrm{SD} 40,3 ; 0-100)$ & 67,4 (SD 41,6; 0-100) & $63,9(\mathrm{SD} 37,6 ; 0-100)$ & 0,068 & 0,93 \\
\hline Sitting & $90,5(\mathrm{SD} 25,3 ; 0-100)$ & 90,4 (SD 24,6; 0-100) & 90,0 (SD 25,9; 0-100) & 0,002 & 1.00 \\
\hline Public transport & $68,4(\mathrm{SD} 68,4 ; 0-100)$ & 60,9 (SD 49,9; 0-100) & $33,3(\mathrm{SD} 48,5 ; 0-100)$ & 2,657 & 0,08 \\
\hline
\end{tabular}

$\mathrm{F}=$ female $; \mathrm{L}=$ left $; \mathrm{THA}=$ Total Hip Arthroplasty $; \mathrm{ASA}=$ American Society of Anesthesiologists score $; \mathrm{F} / \mathrm{U}=$ follow-up VAS = Visual Analogue Scale ; WOMAC = Western. Ontario and McMaster Osteoarthritis Index; EQ-5D = EuroQol 5-Dimensional Health Questionnair

fractures during the period from January 2006 to December 2018. They were matched sequentially against patients of the same sex and age who underwent pTHA or rTHA in the same year.

Thirty-sex patients (23 females, mean age 68,0 y (SD 14,0; 34-86), 24 left hips) who had undergone cTHA were identified (table I). There were 11 failed Dynamic Hip Screws (DHS), 10 HA, 4 cephomedullary nails, 9 cannulated screws, and 2 proximal femoral plates. All pTHAs were performed for OA. Indications for rTHA included a mix of infection, loosening, instability, polyethylene wear, leg length discrepancy and (peri)prosthetic fracture.

Medical records and all available radiographs were reviewed and data was collected on implants used, major complications (DVT/PE, death during admission, dislocation, prosthetic joint infection, periprosthetic fracture, and loosening), mortality after 1 year and at final follow-up. Patients were contacted for an interview over the phone (experienced complications, Western Ontario and 


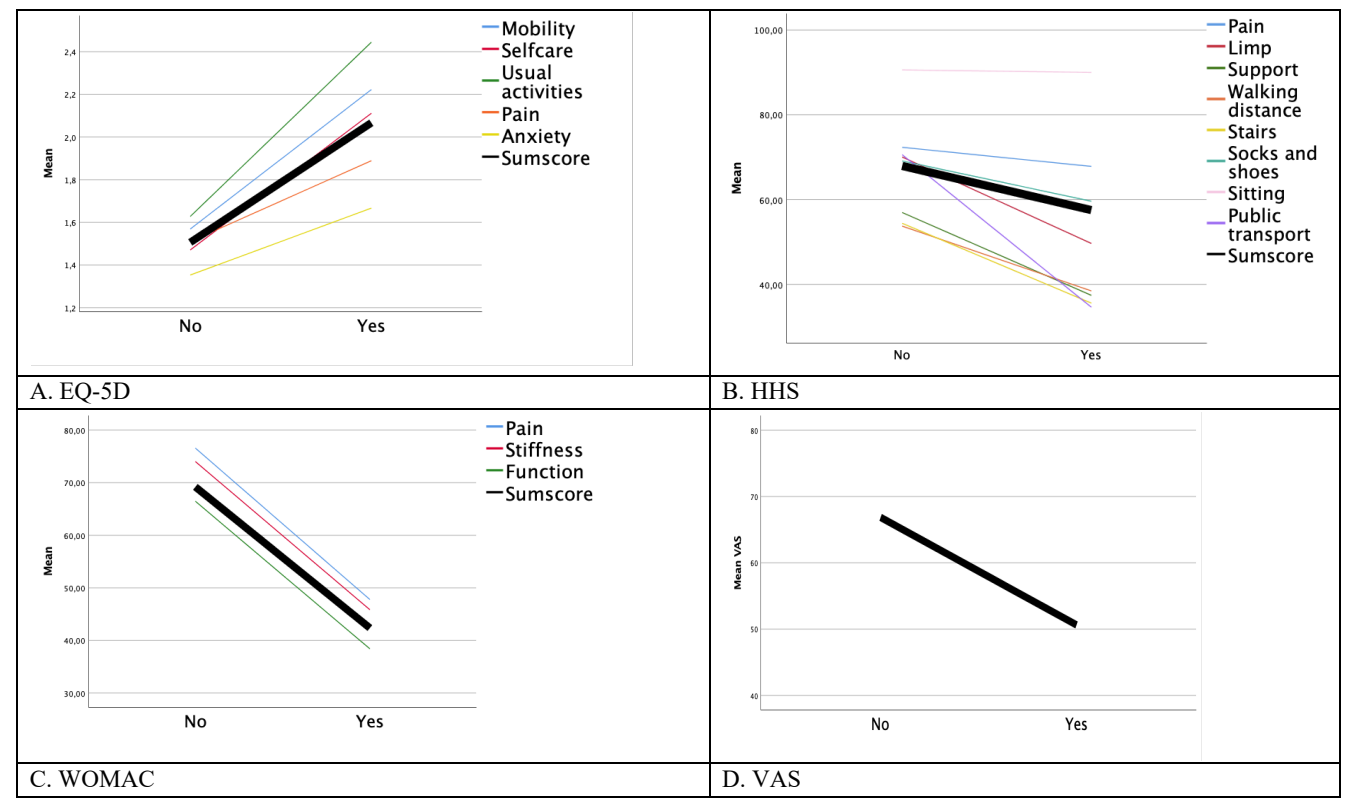

Figure 2. - Multiple line charts of EQ-5D, WOMAC, HHS and VAS scores. No $=$ no revision implants needed and no major complications during follow-up ;

Yes $=$ revision implants needed and/or major complications during follow-up.

McMaster Osteoarthritis Index (WOMAC), Harris Hip Score (HHS), Visual Analogue Scale (VAS) and the EQ-5D Health Questionnaire). If after 4 attempts patients could not be reached, data was considered missing. Standardised sumscores for the WOMAC and domain index scores for the EQ-5D were calculated as per the respective instruction manuals. Total scores for the HHS could not be calculated as information on deformity and mobility was missing for the majority of patients. Therefore, percentages of domain scores were calculated, e.g. if a patient stated he had "marked pain, serious limitation of activities" he scored 10/44 for the pain domain.

Statistical evaluation was performed using IBM Statistical Package for the Social Sciences version 25. One-way ANOVA testing was used to compare means between the three cohorts for ratio and interval variables. If $\mathrm{p} \leq 0.05$ was encountered, subsequent independent sample t-tests were used to see between which groups the statistically significant difference existed. Next, two new cohorts were created, i.e. patients who underwent surgery using primary implants and had an uncomplicated follow-up vs. patients who required revision implants and/or had a complicated follow-up, regardless of this being a pTHA, cTHA, or rTHA. PROMs were compared between these two groups using independent sample t-tests.

This retrospective matched cohort study was recognized as a service improvement project by the institutional review board of the University College London Hospital.

\section{RESULTS}

\section{Hypothesis 1 : The distinction pTHA / cTHA / rTHA will not be able to explain differences in outcomes}

Table I shows the baseline characteristics, mortality and PROMs of the three groups of patients. Cohorts were comparable for age, sex, year of operation, objective (ASA) and subjective (VAS) overall health scores, and duration of follow-up (table I). No statistically significant differences in mortality after one year $(p=0,17)$ or at final followup $(p=0,68)$ were found. Major complications were rare (figure 3D : 14 in total during 691 patients years of follow-up) and did not differ significantly between groups. 


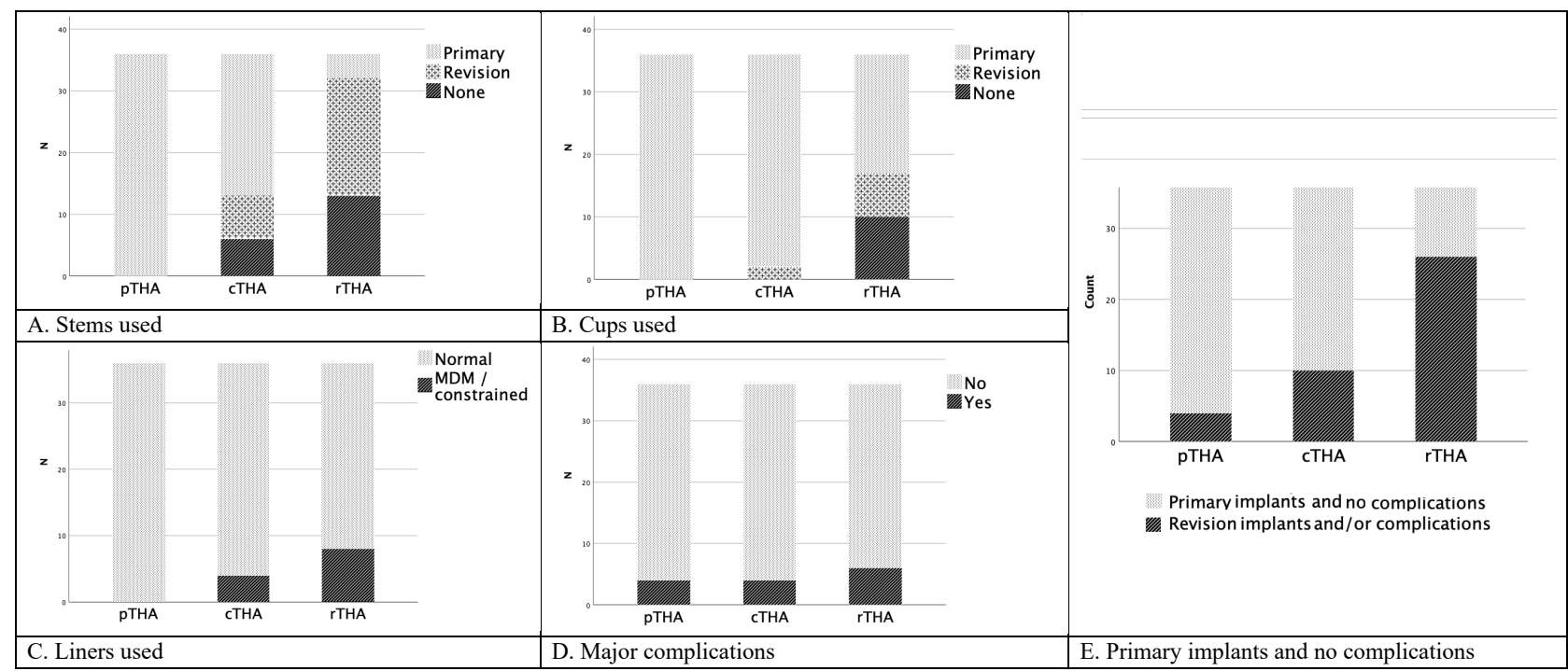

Figure 3. - Stacked bar charts of implants used and major complications.

PROMs were obtained for 19 of the pTHA patients ( 9 deceased, 8 missing), 23 of the cTHA patients (7 deceased, 6 missing), and 18 of the rTHA patients (6 deceased, 12 missing). Standardized WOMAC sumscores, EQ-5D domain index scores, and HHS percentage scores did not show statistically significant differences between the 3 cohorts (Table $1)$.

\section{Hypothesis 2 : Necessity of revision implants and development of major complications will be able to explain differences in outcomes}

Next patients were divided into 2 groups (figure $3 \mathrm{E})$ : patients who did not require revision implants and experienced no major complications during follow-up ("No", $\mathrm{n}=68$ ) and patients who required revision implants and/or experienced major complications during follow-up ("Yes", $\mathrm{n}=40$ ). Figure 2 illustrates the significant differences in all WOMAC sumscores, EQ-5D domain index scores, HHS percentage scores (except "sitting") and VAS general health scores. Therefore, more difficult operations (i.e. the use of revision implants) and setbacks during follow-up (major complications) will influence PROMs.

Hypothesis 3 : Most cTHAs can be performed using primary implants and have an uncomplicated post-operative course
Figure 3 shows the amount of revision stems, revision cups and revision liners (constrained or dual mobility) used for the 3 groups of patients. For all pTHAs primary implants had been used. Significant more revision stems (7 vs $19 ; \mathrm{p}<0.01)$ and revision cups $(2 \mathrm{vs} 7 ; \mathrm{p}<0.01)$ were deemed necessary for rTHA cases compared to cTHA cases. More revision liners were used in the rTHA group than in the cTHA group, but this did not reach statistical significance $(\mathrm{p}=0,21)$. As can be seen in figure $3 \mathrm{E} 26 / 36(72 \%)$ patients who underwent cTHA were treated with primary implants and never suffered from a major complication. As already illustrated in figure 2 PROMs are high in this group of patients.

\section{DISCUSSION}

This study matched and compared a cohort of patients who underwent cTHA to patients who underwent pTHA and rTHA. Several conclusions can be made.

Firstly, the distinction pTHA / cTHA / rTHA is not able to explain differences in outcomes. No significant differences in major complications, mortality or PROMs were found. We therefore propose to stop using this distinction when trying to predict complications, implant survival and costs. The type of primary implant, whether this is a nail, a 
plate, a HA or a THA, is not correlated to the outcome of redo surgery as this will yield very diverse groups and does not take into account more important predicting factors. In the present study, we found that the use of revision implants and the development of major complications could explain almost all differences seen in PROMs. The development and validation of a prediction model based on these and other parameters, e.g. patient frailty, the presence of pre-existent infection/instability, and significant bone loss, would be highly useful in daily clinical practice and for calculation of long-term costs for the society.

Secondly, we were able to perform most cTHAs using primary implants and most of them encountered no major complications during followup. We know, that for this group of patients, costs are low and patient satisfaction is high, comparable to pTHA performed for OA. Other studies have reported similar mid-term results. Archibeck et al. reviewed 102 THA patients after failed fixation of a hip fracture (7). Despite needing slightly more revision type femoral implants (32\% versus $23 \%$ in the present study) they still had excellent outcomes with a mean HHS of 81.8 at last follow-up. Gjertsen et al. found that survival of the implants in the Norwegian Arthroplasty Registry 5 years after cTHA for failed internal fixation of femoral neck fractures was $96 \%$ (18). Most recently, Morsi et al reported on the clinical and radiological outcomes of converting aseptic failures of intertrochanteric fracture fixation using a DHS to a THA in a single stage procedure. Standard straight, polished, collarless, cemented stems were used in all 107 cases. At an average follow-up of 7.4 years they report $99 \%$ implant survivorship, a HHS of 89.3 (range 71-95) and only a very small number of surgical complications (19).

We do realize that all our cTHAs were performed within a high-volume arthroplasty unit with specialist hip surgeons and fellows. Contrary to proximal femur fractures that are ideally treated within 24 hours and therefore often by the oncall team, it is our opinion that cTHAs should be performed by a dedicated hip surgeon, even if this means postponing the procedure.

This study has several limitations. Due to its retrospective nature confounding factors such as patient expectations were not investigated. McLawhorn et al. and Qun et al found that patients who underwent cTHA required more transfusions, had longer operative times and length of hospital stays, and more often had non-home bound discharge $(13,15)$. Due to the absence of these data no cost analysis could be made. Although a period of 13 years was searched, still a relatively small cohort of cTHA patients was found, yet larger than reported in most studies (10-17). Major complications could have been missed and not all patients were reached for questionnaire assessment.

\section{CONCLUSION}

In conclusion, predicting outcome and patient satisfaction based on the fact that the surgical procedure to be performed is a conversion rather than a revision is not useful. Nevertheless, most cTHAs can be performed using primary implants, and most patients report no major complications and high satisfaction.

\section{REFERENCES}

1. Learmonth ID, Young C, Rorabeck C. The operation of the century: total hip replacement. Lancet 2007; 370(9597) : 1508-1519.

2. Rietbergen L, Kuiper JW, Walgrave S, et al. Quality of life after staged revision for infected total hip arthroplasty : a systematic review. Hip Int. 2016 ; 26(4) : 311-318.

3. Brown JM, Mistry JB, Cherian JJ, et al. Femoral Component Revision of Total Hip Arthroplasty. Orthopedics 2016 ; 39(6) : e1129-e1139.

4. Ahmad AQ, Schwarzkopf R. Clinical evaluation and surgical options in acetabular reconstruction: A literature review. J. Orthop 2015 ; 12(Suppl 2) : S238-243.

5. Mahmoud SS, Pearse EO, Smith TO, et al. Outcomes of total hip arthroplasty, as a salvage procedure, following failed internal fixation of intracapsular fractures of the femoral neck : a systematic review and meta-analysis. Bone Joint J. 2016 ; 98-B(4) : 452-460.

6. Lee YK, Kim JT, Alkitaini AA, et al. Conversion Hip Arthroplasty in Failed Fixation of Intertrochanteric Fracture : A Propensity Score Matching Study. J. Arthroplasty 2017 ; 32(5) : 1593-1598.

7. Archibeck MJ, Carothers JT, Tripuraneni KR, et al. Total hip arthroplasty after failed internal fixation of proximal femoral fractures. J. Arthroplasty 2013 ; 28(1) : 168-171. 
8. Krause PC, Braud JL, Whatley JM. Total hip arthroplasty after previous fracture surgery. Orthop. Clin. North Am. $2015 ; 46(2): 193-213$.

9. Franzen H, Nilsson LT, Stromqvist B, et al. Secondary total hip replacement after fractures of the femoral neck. $J$. Bone Joint Surg. Br. $1990 ; 72: 784$.

10. Sarpong NO, Gross MJ, Lakra A, et al. Hemiarthroplasty Conversion : A Comparison to Primary and Revision Total Hip Arthroplasty. J. Arthroplasty 2019 ; 34(6) : 1168-1173.

11. Baghoolizadeh M, Schwarzkopf R. The Lawrence D. Dorr Surgical Techniques \& Technologies Award: Conversion Total Hip Arthroplasty : Is it a Primary or Revision Hip Arthroplasty. J. Arthroplasty 2016 ; 31(9 Suppl) : 16-21.

12. Chin G, Wright DJ, Snir N, et al. Primary vs Conversion Total Hip Arthroplasty: A Cost Analysis. J. Arthroplasty 2016 ; 31(2) : 362-367.

13. McLawhorn AS, Schairer WW, Schwarzkopf R, et al. Alternative Payment Models Should Risk-Adjust for Conversion Total Hip Arthroplasty : A Propensity ScoreMatched Study. J. Arthroplasty. 2018 ; 33(7) : 2025-2030.

14. Mortazavi SM, R Greenky M, Bican O, et al. Total hip arthroplasty after prior surgical treatment of hip fracture is it always challenging? J. Arthroplasty $2012 ; 27(1)$ : 31-36.
15. Qin CD, Helfrich MM, Fitz DW, et al. Differences in Post-Operative Outcome Between Conversion and Primary Total Hip Arthroplasty. J. Arthroplasty 2018 ; 33(5) : 14771480.

16. Ryan SP, DiLallo M, Attarian DE, et al. Conversion vs Primary Total Hip Arthroplasty: Increased Cost of Care and Perioperative Complications. J. Arthroplasty 2018 ; 33(8) : 2405-2411.

17. Schwarzkopf R, Chin G, Kim K, et al. Do Conversion Total Hip Arthroplasty Yield Comparable Results to Primary Total Hip Arthroplasty? J. Arthroplasty 2017 ; 32(3) : 862-871.

18. Gjertsen JE, Lie SA, Fevang JM, et al. Total hip replacement after femoral neck fractures in elderly patients : results of 8,577 fractures reported to the Norwegian Arthroplasty Register. Acta Orthop. 2007 ; 78(4) : 491-497.

19. Morsi EMZ, Drwish AEE, Saber AM, et al. The use of standard cemented femoral stems in total hip replacement after failed internal fixation of intertrochanteric fractures. J. Arthroplasty. $2020 ; 35(9)$ : 2525-2528. 